\title{
Immunomodulatory effect of photodynamic therapy in Galleria mellonella infected with Porphyromonas gingivalis
}

\author{
Jéssica Diane dos Santos*, Janaína Araújo de Alvarenga, Rodnei Dennis Rossoni, \\ Maíra Terra García, Renata Mendonça Moraes, Ana Lia Anbinder, \\ Antonio Olavo Cardoso Jorge, Juliana Campos Junqueira
}

Department of Biosciences and Oral Diagnosis, Institute of Science and Technology, UNESP - Univ. Estadual Paulista, São José dos Campos, SP, Brazil

\section{A R T I C L E I N F O}

\section{Article history:}

Received 21 May 2017

Received in revised form

26 July 2017

Accepted 27 July 2017

Available online 28 July 2017

\section{Keywords:}

Porphyromonas gingivalis

Photodynamic therapy

Galleria mellonella

\begin{abstract}
A B S T R A C T
Porphyromonas gingivalis is an important pathogen in the development of periodontal disease. Our study investigated if the treatment with antimicrobial photodynamic therapy (aPDT) that employs a nontoxic dye, followed by irradiation with harmless visible light can attenuate the experimental infection of $P$. gingivalis in Galleria mellonella. Firstly, different concentrations of $P$. gingivalis ranging from $10^{2}$ to $10^{6}$ cells/larva were injected into the animal to obtain a lethal concentration. Next, the following groups of G. mellonella infected with $P$. gingivalis were evaluated: inoculation of the photosensitizer and application of laser $(\mathrm{P}+\mathrm{L}+)$, inoculation of physiologic solution and application of laser $(\mathrm{P}-\mathrm{L}+)$, inoculation the photosensitizer without laser $(\mathrm{P}+\mathrm{L}-)$ and inoculation of physiologic solution without Laser (P-L-). The effects of aPDT on infection by $P$. gingivalis were evaluated by survival curve analysis and hemocytes count. A lethal concentration of $10^{6}$ cells/larva was adopted for evaluating the effects of aPDT on experimental infection with $P$. gingivalis. We found that after $120 \mathrm{~s}$ of PDT application, the death of G. mellonella was significantly lower compared to the control groups $(p=0.0010)$. Moreover, the hemocyte density in the $\mathrm{P}+\mathrm{L}+$ group was increased by $9.6 \times 10^{6}$ cells $/ \mathrm{mL}(2.62$-fold increase) compared to the infected larvae with no treatment (L-P-group) $(p=0.0175)$. Finally, we verified that the aPDT led to a significant reduction of the number of $P$. gingivalis cells in $G$. mellonella hemolymph. In conclusion, PDT application was effective against $P$. gingivalis infection by increasing the survival of $G$. mellonella and was able to increase the circulating hemocytes indicating that PDT activates the G. mellonella immune system.
\end{abstract} (c) 2017 Elsevier Ltd. All rights reserved.

\section{Introduction}

Hundreds of bacterial species that colonize the oral cavity maintain the balance of the ecosystem protecting against the invasion by others pathogenic species. Although some of these species can become pathogenic in response to the host genotype, stress, diet or behavior (e.g. smoking) [1-5]. Periodontal diseases are among the most common chronic inflammatory diseases in humans and are associated with the presence of Porphyromonas gingivalis. This oral bacterial specie is an anaerobic Gram-negative coccobacillus, which belongs to the Bacteroidaceae family, present colonies black-colored in blood agar and requires anaerobic

\footnotetext{
* Corresponding author. Department of Biosciences and Oral Diagnosis, Institute of Science and Technology, UNESP - Univ. Estadual Paulista, Francisco José Longo 777, São Dimas, São José dos Campos, CEP: 12245-000, SP, Brazil.

E-mail address: jessica.santos@ict.unesp.br (J.D. dos Santos).
}

conditions for its growth. This microorganism obtains its metabolic energy through the fermentation of aminoacids, an important property for its survival in deep periodontal pockets, where carbohydrates are scarce [6,7].

$P$. gingivalis is described as a "keystone pathogen" in chronic periodontitis. In the oral cavity, this species is able to deregulate the host immune response to favor the biofilm formation, thereby interrupting homeostasis with the host causing dysbiosis and local disease [8]. The pathogenicity of $P$. gingivalis is attributed to a number of virulence factors associated with its surface that include cysteine proteinases (gingipains), fimbriae, haem-binding proteins, and outer membrane transport proteins. Specially, cysteine endoproteinases, Arg-specific gingipains (RgpA and RgpB) and Lys specific gingipain (Kgp) have multiple effects on both innate and adaptive immune responses in the host $[9,10]$. Virulence factors, such as fimbriae and gingipans, are important for the identification, in typing methods, of strains associated with the disease [11]. 
The conventional treatment for periodontitis involves the mechanical removal of dental plaque and mineralized deposits from tooth surfaces, but Antimicrobial photodynamic therapy (aPDT) has been proposed as an adjunctive strategy for periodontitis treatments [12-14]. One of the main advantages of laser is its ability to kill subgingival bacteria in seconds, which minimizes the need for maintaining high concentration of antimicrobial agents within the lesion for a long time [15]. Many oral bacteria are susceptible to the application of laser in the presence of photosensitizers, such as toluidine blue $\mathrm{O}$, methylene blue and malachite green. These findings suggest that aPDT could be potentially advantageous in periodontal therapy [16].

In the last decades, several invertebrate models have been used in the study of the microorganism pathogenicity, host-pathogen interaction and as screening for testing new therapies [17]. The main invertebrate models of experimental infection are a Galleria mellonella, Drosophila melanogaster and the nematode Caenorhabditis elegans [18-20]. G. mellonella presents numerous advantages over other invertebrate models as a sufficient size for the injection and the ability to survive at temperatures ranging from 25 to $37^{\circ} \mathrm{C}$, simulating the natural environment of mammalian hosts. Moreover, this insect have an immune system exhibits both humoral and cellular components; Hemocytes are the major mediator of cellular defenses, which perform similar functions to human macrophages and neutrophils [21-23].

Since in vivo studies are crucial for the evaluation of the microorganism pathogenicity and development of alternative treatments, the aim of this study was to verify the effectiveness of aPDT against experimental infection by $P$. gingivalis in $G$. mellonella model.

\section{Materials and methods}

\subsection{Microbial strain and culture conditions}

Porphyromonas gingivalis (ATCC 33277) strain used in this study was stored at $-80{ }^{\circ} \mathrm{C}$ and cultured on blood agar plates with Fastidious anaerobic agar base (FAA, Acumedia, Michigan, USA) enriched with $1 \%$ menadione (Sigma-Aldrich, São Paulo, Brazil) and hemin (Sigma-Aldrich, São Paulo, Brazil) and incubated in anaerobic jars (Permution, Paraná, Brazil) for $5-7$ days at $37^{\circ} \mathrm{C}$.

\subsection{Preparation of the invertebrate model of Galleria mellonella}

G. mellonella created in the laboratory of Microbiology and Immunology of the Institute of Science and Technology of São José dos Campos - UNESP, in the final instar larval stage were used in this study. Sixteen randomly chosen G. mellonella larvae with similar weight and size $(250-350 \mathrm{mg})$ were used per group in all assays. Two control groups were included in the assays that form part of this study: one group were inoculated with PBS to enable us to observe the demise of the larvae due to physical trauma, and the other received no injection as a control for general viability. A $10 \mu \mathrm{l}$ Hamilton syringe (Hamilton Inc, EUA) was used to inject $10 \mu \mathrm{l}$ inoculum aliquots into the hemocoel of each larvae via the last left proleg. After injection, larvae were incubated at $37{ }^{\circ} \mathrm{C}$ in plastic containers.

\subsection{Survival curve analysis}

For analysis of virulence in G. mellonella and determination of the concentration that was used in the experiments of photodynamic therapy, standardized suspensions was adjusted in physiological solution (PBS) between $10^{2}$ to $10^{6}$ cells/larva by spectrophotometer (B582, Micronal, São Paulo, Brazil) at the wavelength of $660 \mathrm{~nm}$. After injection, larvae were stored in plastic containers at $37^{\circ} \mathrm{C}$ and observed every $24 \mathrm{~h}$ for a period of 7 days, and considered dead when they displayed no movement in response to touch. All experiments were repeated at least twice, and representative experiments are presented. For the survival curve analysis, 16 randomly chosen larvae were used per group in a total of 208 larvae.

\subsection{Photodynamic therapy}

For this study, the methodology described by Chibebe et al. [24] was used with some modifications. The phenothiazinium salt methylene blue (MB, Sigma Aldrich, São Paulo, Brazil) was used as the photosensitizer in this study. MB solutions at a final working concentration of $600 \mathrm{mM}$ were prepared by dissolving the dye in physiological solution. A new photosensitizer solution was prepared on the same day of each experiment. After the photosensitizer injection, larvae were maintained in the dark until the time of light irradiation.

G. mellonella larvae were irradiated in a 24-well culture plate (Costar Corning, New York, NY, USA) as shown in Fig. 1. Light source of Gallium and Aluminum Arsenide laser with wavelength of $660 \mathrm{~nm}$ (visible red), power of $50 \mathrm{~mW}$, with energy density of $15 \mathrm{~J} /$ $\mathrm{cm}^{2}$ was used for light delivery. The experiments were performed as follows: G. mellonella received the photosensitizer injection (10 $\mu \mathrm{L}) 90 \mathrm{~min}$ after the bacterial infection. We waited for at least 30 additional min after the photosensitizer injection to allow a good dispersion of the photosensitizer into the insect body, prior to the light irradiation.

\subsection{Quantification of G. mellonella hemocyte}

Larvae were infected with $P$. gingivalis $\left(10^{6}\right.$ cells/larvae) by injecting the bacteria at the last left proleg and submitted to PDT as described above. After the larvae were incubated at $37^{\circ} \mathrm{C}$ for $4 \mathrm{~h}$. At each time point, the larvae were cut in the cephalocaudal direction with a scalpel blade and squeezed to remove the hemolymph, which was transferred to an Eppendorf tube. The tubes contained cold, sterile insect physiologic saline (IPS) (150 mM sodium chloride; $5 \mathrm{mM}$ potassium chloride; $100 \mathrm{mM}$ Tris-hydrochloride, $\mathrm{pH} 6.9$

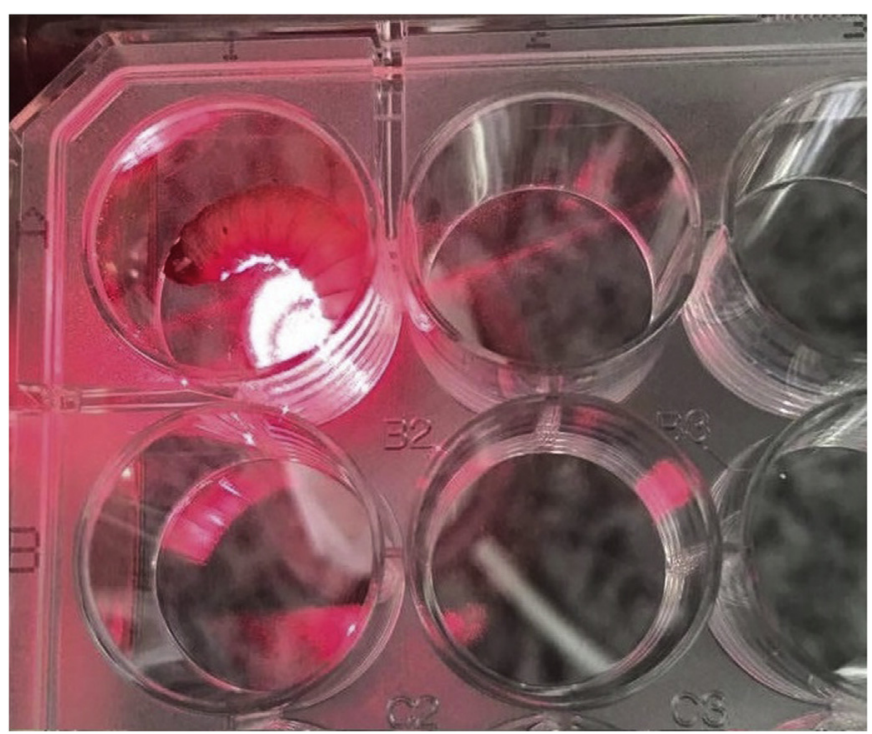

Fig. 1. In vivo photosensitization. G. mellonella being subjected to light irradiation. Each larvae was irradiated in a separate well of a 24-well culture plate. 
with $10 \mathrm{mM}$ EDTA, and $30 \mathrm{mM}$ sodium citrate). The hemocytes were enumerated with the aid of a hemocytometer; however, we did not differentiate between the 6 types of hemocytes. The results were averaged from four replicates. For the quantification of G. mellonella hemocyte, we used 16 larvae per group in a total of 128 larvae.

\subsection{Persistence of P. gingivalis in the hemolymph}

The number of bacterial cells in the hemolymph was measured at 0,4 and $8 \mathrm{~h}$ after larvae were irradiated in a 24-well culture plate. At each indicated time-point, 9 surviving larvae per group were bled by insertion of a lancet into the hemocoel. Hemolymph from 9 larvae was pooled into $1.5 \mathrm{ml}$ Eppendorf tubes in a final volume of approximately $130 \mu \mathrm{L}$. Then, the hemolymph was homogenized, serially diluted, and plated on blood agar plates with Fastidious anaerobic agar base (FAA, Acumedia, Michigan, USA) enriched with 1\% menadione (Sigma-Aldrich, São Paulo, Brazil) and hemin (Sigma-Aldrich, São Paulo, Brazil). Plates were incubated in anaerobic jars (Permution, Paraná, Brazil) for $5-7$ days at $37{ }^{\circ} \mathrm{C}$, and colonies were counted in each pool (CFU/pool). For the quantification of CFU of $P$. gingivalis in the hemolymph, we used 9 larvae per group in a total of 54 larvae.

\subsection{Statistical analysis}

Survival curves were constructed by the Kaplan Meier method and compared by the Log-rank (Mantel-Cox) test. Analysis of variance (ANOVA) and Tukey test were used to compare the results obtained in the data of hemocyte count. All the tests were performed using GraphPad Prism statistical software (GraphPad Software, Inc., California, CA, USA) and aPvalue0.05 was considered significant.

\section{Results}

Initially, a set of assays was performed to provide a comprehensive understanding of the host response following $P$. gingivalis infection in $G$. mellonella model and to determine the lethal inoculum concentration. We tested concentrations ranging from $10^{2}$ to $10^{6}$ cells/larva and observed death in most of the larvae at the concentrations of $10^{4}, 10^{5}$ and $10^{6}$ cells/larvae (Fig. 2). Based on these results, a lethal concentration of $10^{6}$ cells/larva was adopted for the study to determine the effects of PDT on experimental infection with $P$. gingivalis.

After we asked whether this host-pathogen system could treated with an alternative treatment like PDT. We used methylene

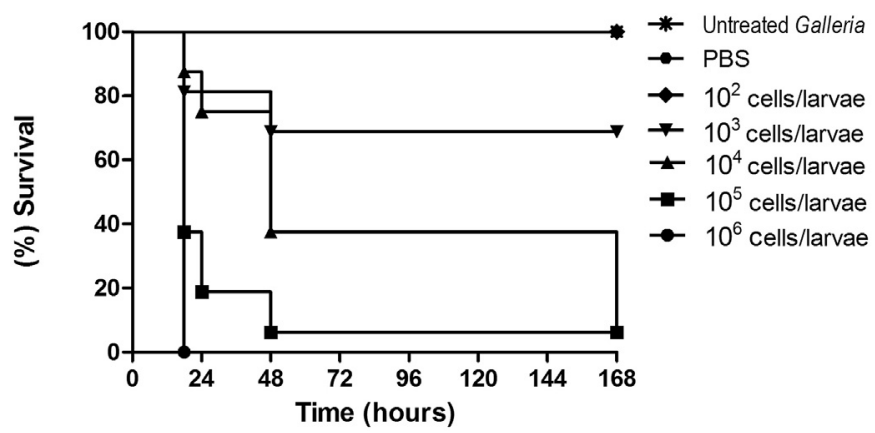

Fig. 2. Killing of G. mellonella larvae by P. gingivalis. G. mellonella larvae were injected with serial concentrations of different $P$. gingivalis (cells/larva). The control groups were composed of untreated G. mellonella larvae that received only PBS injection and one group with no injection.

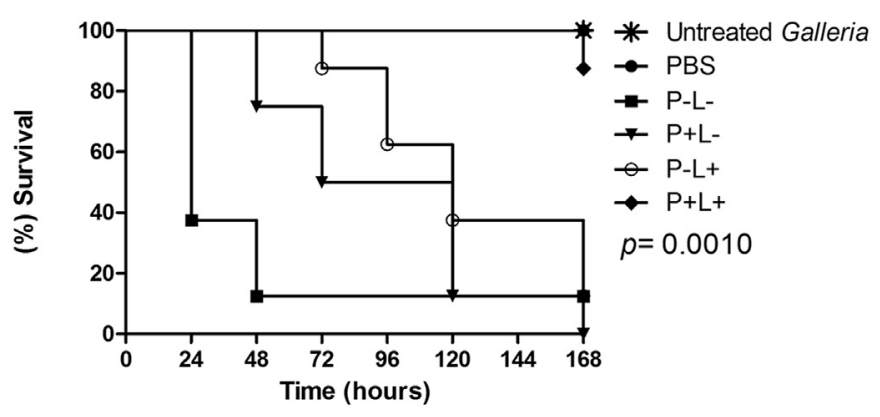

Fig. 3. PDT as alternative treatment prolongs the survival of $G$. mellonella larvae infected with $P$. gingivalis. There was a significant difference between the $\mathrm{P}+\mathrm{L}+$ and P-L- control group $(p=0.0010)$. Larvae exhibited survival for $75 \%$ of the treatment with PDT compared to the infected larvae without treatment. Log-rank test, $\mathrm{p} \leq 0.05$.

blue as photosensitizer because according Chibebe et al. [24] it is reported low toxicity and broad clinical applicability. Moreover, this photosensitizer did not cause melanization or death. After irradiation, the survival rate of $G$. mellonella was counted $24 \mathrm{~h}$ post $P$. gingivalis infection. We found that after $120 \mathrm{~s}$ of PDT application, the death of $G$. mellonella was significantly lower compared to the control groups $(p=0.0010)$ indicating that the PDT was efficient in combating $P$. gingivalis infection (Fig. 3 ). The other control groups $\mathrm{P}+\mathrm{L}-(p=0.0683)$ and $\mathrm{P}-\mathrm{L}+(p=0.0528)$ did not have a statistically significant difference compared to the P-L- group (P. gingivalis control group).

Additionally, we investigated the ability of PDT to prime the G. mellonella immune response by evaluating changes in the number of available hemocytes. As it was verified a higher survival rate of the larvae with the PDT group $(\mathrm{P}+\mathrm{L}+)$ compared to the $P$. gingivalis control group (L-P-), we tested whether this alternative treatment could affect the hemocyte density $4 \mathrm{~h}$ post-injection. $P$. gingivalis (P-L- group) reduced the number of hemocytes compared to the PBS group but there was no significant difference between these groups $(p=0.3042)$. However, the hemocyte density in the PDT group $(\mathrm{P}+\mathrm{L}+)$ was increased by $9.6 \times 10^{6}$ cells $/ \mathrm{mL}$ (2.62-fold increase) compared to the infected larvae with no treatment (L-P-group) ( $p=0.0175$; Fig. 4). These data suggest that PDT has some immunomodulatory effect in the G. mellonella model. In this assay, we performed only the PBS and L-P- as control groups

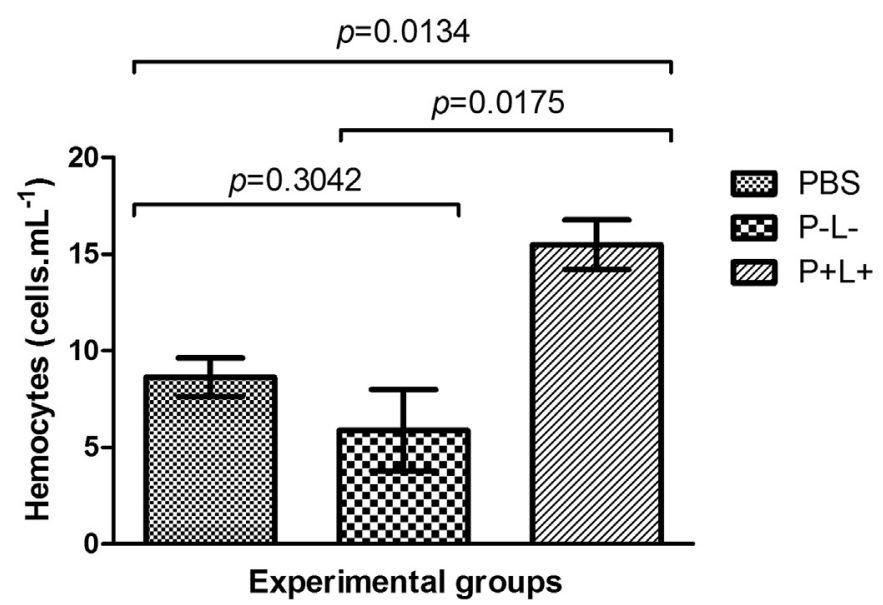

Fig. 4. The G. mellonella hemocyte density increased with the PDT treatment. The hemolymph of larvae treated with PDT was collected to determine the hemocyte density compared with the control groups. Student's t-test was used to compare hemocyte densities between the experimental groups. A p value $\leq 0.05$ was considered significant. 


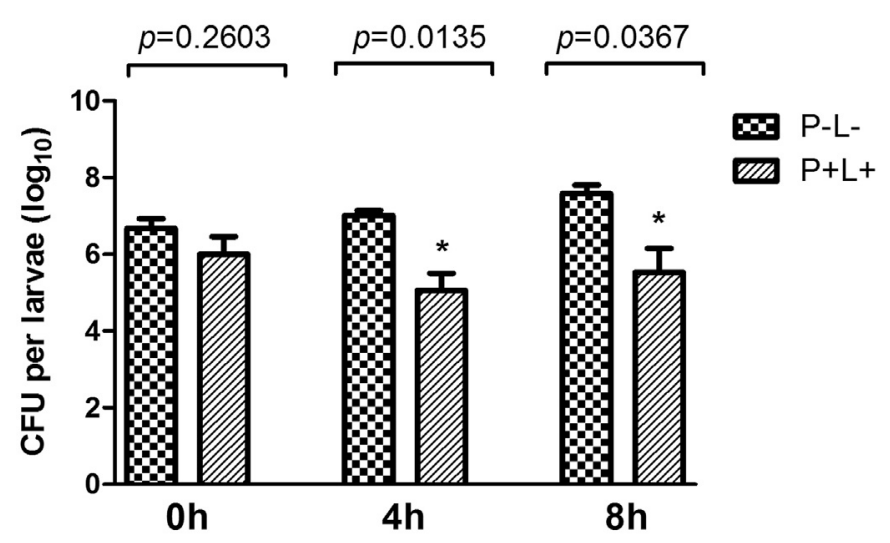

Fig. 5. PDT decreased the number of $P$. gingivalis in $G$. mellonella hemolymph. Mean and standard deviation of $P$. gingivalis counts (CFU/larvae) in the hemolymph of Galleria mellonella after 0, 4 and $8 \mathrm{~h}$ of PDT application. The following groups were compared at each time: P-L- (control) and P + L+ (PDT group). Significant differences were observed after 4 and $8 \mathrm{~h}$ of PDT application, with a larger number of CFU/larvae in the control group compared to the $\mathrm{P}+\mathrm{L}+$ group ( $4 \mathrm{~h}: p=0.0135 ; 8 \mathrm{~h}: p=0.0367$ ). Student $t$-test, $\mathrm{p} \leq 0.05$.

since in the survival curve the P $+\mathrm{L}$ - (Photosensitizer only) and P$\mathrm{L}+$ (Laser only) had no increase the survival of the larvae.

The study of $G$. mellonella hemolymph culture revealed lower growth of $P$. gingivalis in the PDT group $(\mathrm{P}+\mathrm{L}+)$ compared to $P$. gingivalis control group (P-L-) at the evaluated times, confirming the results obtained in the survival tests and hemocyte counting. A significant difference between groups was observed at 4 and $8 \mathrm{~h}$ after the application of PDT, with higher growth of $P$. gingivalis in the control group (7.58 Log) compared to the PDT group (5.52 Log) (Fig. 5).

\section{Discussion}

Mammalian animal models are important tools for the evaluation of microbial virulence factors. However, invertebrate models are increasingly being used as a viable alternative to traditional animal models $[25,26]$. In this respect, G. mellonella has been found to be an interesting invertebrate model for the study of important human pathogens, including $P$. gingivalis and to evaluate alternative treatments such as PDT. The efficacy of PDT consists of irradiating a photosensitive agent with a light source of suitable wavelength in the presence of oxygen to produce reactive oxygen species or free radicals that damage cells [27].

First, a range of the initial $P$. gingivalis inoculum was injected in G. mellonella at concentrations of $10^{2}, 10^{3}, 10^{4}, 10^{5}$ and $10^{6} \mathrm{CFU} /$ larva resulting in $0,32,93,93$ and $100 \%$ of mortality, respectively, after $168 \mathrm{~h}$ of infection. Similar result was found with Gomes et al. [28] who evaluated the antimicrobial activity of pomegranate glycolic extract in infected G. mellonella with $10^{4}$ cells/larvae of $P$. gingivalis. $P$. gingivalis pathogenicity is related to the production of virulence factors including biofilm formation, several varieties of lipopolysaccharide and a family of proteases known as the gingipains, many of which play a role in the development and progression of periodontal disease $[29,30]$. In addition, in rodent models this bacteria has been associated with promoting a dysbiotic micro-flora and propagating a hyper-inflammatory process [31,32].

The PDT application on G. mellonella infected was effective promoting a significant $75 \%$ larvae survival rate in PDT exposed group compared to the control group P-L-. The other groups (P + Land $\mathrm{P}-\mathrm{L}+$ ) did not promote an increase in the survival of the animals. Agreeing with our results, Chibebe et al. [33] also found that PDT application prolonged the survival of $C$. albicans infected G. mellonella larvae and consequently reducing the fungal burden on their hemolymph. The use of Galleria is very important in the standardization of new protocols being suitable as a screening for the study in rodent models that are considered "gold standard models" in the pathogen-host studies.

According Chibebe et al. [24] the increase of larvae survival can be explained because PDT in G. mellonella dose should promotes bacterial cell-wall damage, thus facilitating the insect immune system response to solve the infection. With a permeable cell wall, bacteria could become easily phagocytized by G. mellonella hemocytes, and are more susceptible to humoral insect immune response, by antimicrobial peptide action.

Based on these results, we investigated the capacity of PDT to stimulate the immune system of $G$. mellonella. It was observed that the hemocyte density in the PDT group was increased $9.6 \times 10^{6}$ cells $/ \mathrm{mL}$ compared to the infected larvae with no treatment; these findings indicate that PDT is capable to stimulate the cellular immune response of the larvae accompanied by an increase in the number of hemocytes. Interestingly, we also observed that the P-L- group showed a reduction of hemocyte numbers in relation to the PBS control group, indicating that $P$. gingivalis suppresses the hemocyte count. According Bergin et al. [34] and Rossoni et al. [35] the level of hemocytes cells can be used to determine the pathogenicity of microorganisms, modulations of the immune response and consequently check the status of health and/or disease of larvae.

Since we know the quantity of $P$. gingivalis injected directly into the hemolymph of larvae, we also evaluated the effects of PDT on $P$. gingivalis cells present in the hemolymph of $G$. mellonella at different times after irradiation $(0,4$ and $8 \mathrm{~h})$. The results showed that the PDT affect the number of $P$. gingivalis in the hemolymph at the times 4 and $8 \mathrm{~h}$. Agreeing with our results, Chibebe et al. [24] investigated the action of PDT on different strains of $E$. faecium in G. mellonella model. After photosensitization of larvae, the survival of the insects increased and the bacterial burden on the hemolymph at the times of 4 and $8 \mathrm{~h}$ after the PDT decreased. These results indicate that the PDT can be an alternative for the treatment of infectious diseases and G. mellonella is suitable to perform PDTbased protocols in periodontal pathogens.

To the best of our knowledge, no studies have investigated the PDT application on infected $G$. mellonella with $P$. gingivalis. These promising results show that $G$. mellonella is a suitable model for the study of periodontal pathogens as well as alternative therapies, indicating the importance of further studies employing different assays (phagocytosis, analysis of antimicrobial peptide production and tissue cultures) that may allow analyzing both host and microorganism in order to elucidate characteristics of $P$. gingivalis virulence, like its capacity of inducing a persistent periodontal infection.

Within the limits of this study, it may be concluded that the PDT application was effective against $P$. gingivalis infection by increasing the survival of $G$. mellonella larvae. Moreover, this study indicates that PDT activates the G. mellonella immune system by an increase of circulating hemocytes.

\section{Acknowledgements}

This study was supported by the state funding agency Fundação de Amparo à Pesquisa do Estado de São Paulo (FAPESP), Brazil (grant 2013/26820-4).

\section{References}

[1] A. Al-Rasheed, H. Scheerens, D.M. Rennick, H.M. Fletcher, D.N. Tatakis, Accelerated alveolar bone loss in mice lacking interleukin-10, J. Dent. Res. 82 (2003) 632-635. 
[2] J.A. Aas, B.J. Paster, L.N. Stokes, I. Olsen, F.E. Dewhirst, Defining the normal bacterial flora of the oral cavity, J. Clin. Microbiol. 43 (2005) 5721-5732.

[3] P. Belda-Ferre, L.D. Alcaraz, R. Cabrera-Rubio, H. Romero, A. Simon-Soro, M. Pignatelli, A. Mira, The oral metagenome in health and disease, ISME J. 6 (1) (2012) 46-56, http://dx.doi.org/10.1038/ismej.2011.85.

[4] C.L. Almeida-da-Silva, A.C. Morandini, H. Ulrich, D.M. Ojcius, R. Coutinho-Silva, Purinergic signaling during Porphyromonas gingivalis infection, Biomed. J. 39 (4) (2016 Aug) 251-260, http://dx.doi.org/10.1016/j.bj.2016.08.003. Epub 2016 Sep. 24.

[5] G. Reynolds-Campbell, A. Nicholson, C.A. Thoms-Rodriguez, Oral bacterial infections: diagnosis and management, Dent. Clin. North Am. 61 (2) (2017 Apr) 305-318, http://dx.doi.org/10.1016/j.cden.2016.12.003.

[6] P.E. Kolenbrander, R.J. Palmer Jr., S. Periasamy, N.S. Jakubovics, Oral multispecies biofilm development and the key role of cell-cell distance, Nat. Rev. Microbiol. 8 (2011) 471-480.

[7] V. Zijnge, M.B. van Leeuwen, J.E. Degener, F. Abbas, T. Thurnheer, R. Gmur, H.J. Harmsen, Oral biofilm architecture on natural teeth, PLoS One 5 (2011) e9321.

[8] G. Hajishengallis, R.P. Darveau, M.A. Curtis, The keystone-pathogen hypothesis, Nat. Rev. Microbiol. 10 (2012) 717-725, 10.1038/nrmicro2873.

[9] K. Popadiak, J. Potempa, K. Riesbeck, A.M. Blom, Biphasic effect of gingipains from Porphyromonas gingivalis on the human complement system, J. Immunol. 178 (11) (2007 Jun 1) 7242-7250.

[10] N. Bostanci, G.N. Belibasakis, Porphyromonas gingivalis: an invasive and evasive opportunistic oral pathogen, FEMS Microbiol. Lett. 333 (2012) 1-9.

[11] T. Yoshino, M.L. Laine, A.J. Van Winkelhoff, G. Dahlen, Genotype variation and capsular serotypes of Porphyromonas gingivalis from chronic periodontitis and periodontal abscesses, FEMS Microbiol. Lett. (2007) 270.

[12] I.M. Diniz, I.D. Horta, C.S. Azevedo, T.R. Elmadjian, A.B. Matos, M.R. Simionato, M.M. Marques, Antimicrobial photodynamic therapy: a promise candidate for caries lesions treatment, Photodiagnosis Photodyn. Ther. 12 (3) (2015 Sep) $511-518$.

[13] A.S. Garcez, S.C. Núñez, N. Azambuja, E.R. Fregnani, H.M.H. Rodriguez, M.R. Hamblin, et al. Effects of photodynamic therapy on Gram-positive and Gram negative bacterial biofilms by bioluminescence imaging and scanning electron microscopic analysis, Photomed. Laser Surg. 31 (11) (2013) 519-525.

[14] Y. Sasaki, J.I. Hayashi, T. Fujimura, Y. Iwamura, G. Yamamoto, E. Nishida, T. Ohno, K. Okada, H. Yamamoto, T. Kikuchi, A. Mitani, New irradiation method with indocyanine green-loaded nanospheres for inactivating periodontal pathogens, Int. J. Mol. Sci. 18 (1) (2017 Jan 13) 154.

[15] S. Mathur, S.V. Kothiwale, B.P. Nag T. Mathur, A. Bhansali, R.K. Khatri, Effect of gallium-arsenic laser on photosensitized periodontopathic anaerobic organisms: an in vitro study, J. Indian Soc. Periodontol. 20 (4) (2016 Jul) 369.

[16] F. Sgolastra, A. Petrucci, M. Severino, F. Graziani, R. Gatto, A. Monaco, Adjunctive photodynamic therapy to non-surgical treatment of chronic periodontitis: a systematic review and meta-analysis, J. Clin. Periodontol. 40 (5) (2013) 514-526.

[17] L.E. Maekawa, R.D. Rossoni, J.O. Barbosa, A.O. Jorge, J.C. Junqueira, M.C. Valera Different extracts of Zingiber officinale decrease Enterococcus faecalis infection in Galleria mellonella, Braz Dent. J. 26 (2) (2015 Mar-Apr) 105-109, http:// dx.doi.org/10.1590/0103-6440201300199.

[18] M. Arvanitis, J. Glavis-Bloom, E. Mylonakis, Invertebrate models of fungal infection, Biochim. Biophys. Acta (BBA)-Mol Basis Dis. 1832 (9) (2013 Sep 30) 1378-1383.

[19] H.F. Yang, A.J. Pan, L.F. Hu, Y.Y. Liu, J. Cheng, Y. Ye, J.B. Li, Galleria mellonella as an in vivo model for assessing the efficacy of antimicrobial agents against Enterobacter cloacae infection, J. Microbiol. Immunol. Infect. 50 (1) (2014) $55-61$.

[20] N. Trevijano-Contador, O. Zaragoza, Galleria mellonella: an invertebrate model to study pathogenicity in correctly defined fungal species, Fungal Biol. 120 (2)
(2016) 288-295

[21] S. Barnoy, H. Gancz, Y. Zhu, C.L. Honnold, D.V. Zurawski, M.M. Venkatesan, The Galleria mellonella larvae as an in vivo model for evaluation of Shigella virulence, Gut Microbes 8 (2017 Mar) 1-6.

[22] N. Velikova, K. Kavanagh, J.M. Wells, Evaluation of Galleria mellonella larvae for studying the virulence of Streptococcus suis, BMC Microbiol. 16 (1) (2016 Dec 15) 291.

[23] H.F. Yang, A.J. Pan, L.F. Hu, Y.Y. Liu, J. Cheng, Y. Ye, J.B. Li, Galleria mellonella as an in vivo model for assessing the efficacy of antimicrobial agents against Enterobacter cloacae infection, J. Microbiol. Immunol. Infect. 50 (1) (2014 Nov) 55-61.

[24] J. Chibebe Junior, B.B. Fuchs, C.P. Sabino, J.C. Junqueira, A.O. Jorge, M.S. Ribeiro, M.S. Gilmore, L.B. Rice, G.P. Tegos, M.R. Hamblin, E. Mylonakis, Photodynamic and antibiotic therapy impair the pathogenesis of Enterococcus faecium in a whole animal insect model, PLoS One 8 (2) (2013) e55926, http://dx.doi.org/ 10.1371/journal.pone.0055926.

[25] J.C. Junqueira, Galleria mellonella as a model host for human pathogens: recent studies and new perspectives, Virulence 3 (6) (2012 Oct 1) 474-476, http:// dx.doi.org/10.4161/viru.22493.

[26] C.J. Tsai, J.M. Loh, T. Proft, Galleria mellonella infection models for the study of bacterial diseases and for antimicrobial drug testing, Virulence 7 (3) (2016 Apr 2) 214-229, http://dx.doi.org/10.1080/21505594.2015.1135289.

[27] K. Plaetzer, B. Krammer, J. Berlanda, F. Berr, T. Kiesslich, Photophysics and photochemistry of photodynamic therapy: fundamental aspects, Lasers Med. Sci. 24 (2) (2009 Mar) 259-268, http://dx.doi.org/10.1007/s10103-008-05391.

[28] L.A. Procópio, L.M. Figueiredo, A.L. do Rosário, B.M. Palma, K.C. Castro L. Ruano, A.O. de Oliveira Fugisaki, L.D. Jorge, J.C. Junqueira, Punica granatum L. (pomegranate) extract: in vivo study of antimicrobial activity, Sci. World J. (2016), http://dx.doi.org/10.1155/2016/8626987.

[29] R.J. Lamont, G. Hajishengallis, Polymicrobial synergy and dysbiosis in inflammatory disease, Trends Mol. Med. 21 (3) (2015 Mar) 172-183, http:// dx.doi.org/10.1016/j.molmed.2014.11.004.

[30] E. Connolly, E. Millhouse, R. Doyle, S. Culshaw, G. Ramage, G.P. Moran, The Porphyromonas gingivalis hemagglutinins HagB and HagC are major mediators of adhesion and biofilm formation, Mol. Oral Microbiol. 32 (1) (2017 Feb) 35-47, http://dx.doi.org/10.1111/omi.12151.

[31] G. Hajishengallis, S. Liang, M.A. Payne, A. Hashim, R. Jotwani, M.A. Eskan, M.L. McIntosh, A. Alsam, K.L. Kirkwood, J.D. Lambris, R.P. Darveau, M.A. Curtis, Low-abundance biofilm species orchestrates inflammatory periodontal disease through the commensal microbiota and complement, Cell Host Microbe 10 (5) (2011 Nov 17) 497-506, http://dx.doi.org/10.1016/j.chom.2011.10.006.

[32] S.A. Castro, R. Collighan, P.A. Lambert, I.H. Dias, P. Chauhan, C.E. Bland, I. Milic, M.R. Milward, P.R. Cooper, A. Devitt, Porphyromonas gingivalis gingipains cause defective macrophage migration towards apoptotic cells and inhibit phagocytosis of primary apoptotic neutrophils, Cell Death Dis. 8 (3) (2017 Mar 2) e2644, http://dx.doi.org/10.1038/cddis.2016.481.

[33] J. Chibebe Junior, C.P. Sabino, X. Tan, J.C. Junqueira, Y. Wang, B.B. Fuchs, A.O. Jorge, G.P. Tegos, M.R. Hamblin, E. Mylonakis, Selective photoinactivation of Candida albicans in the non-vertebrate host infection model Galleria mellonella, BMC Microbiol. 13 (2013 Oct 1) 217, http://dx.doi.org/10.1186/14712180-13-217.

[34] D. Bergin, M. Brennan, K. Kavanagh, Fluctuations in haemocyte density and microbial load may be used as indicators of fungal pathogenicity in larvae of Galleria mellonella, Microbes Infect. 5 (15) (2003 Dec) 1389-1395.

[35] R.D. Rossoni, B.B. Fuchs, P.P. de Barros, M.D. Velloso, A.O. Jorge, J.C. Junqueira, E. Mylonakis, Lactobacillus paracasei modulates the immune system of Galleria mellonella and protects against Candida albicans infection, PLoS One 12 (3) (2017 Mar 7) e0173332, http://dx.doi.org/10.1371/journal.pone.0173332. 